\title{
A inserção do profissional de educação física nas equipes multiprofissionais da estratégia saúde da família
}

\author{
Laísa Xavier Schuh¹, Caroline Brand², Suzane Beatriz Frantz Krug³, \\ Edna Linhares Garcia ${ }^{4}$, Anelise Reis Gaya ${ }^{5}$, Maria Amélia Roth ${ }^{6}$
}

\begin{abstract}
RESUMO
O presente estudo objetivou refletir sobre a importância de equipes multiprofissionais na Estratégia Saúde da Família, com enfoque na importância do profissional de Educação Física. A metodologia adotada consistiu de pesquisas na literatura existente, tendo como base a Portaria Ministerial 154 de janeiro de 2008 que criou os Núcleos de Apoio à Saúde da Família (NASFs) e suas diretrizes. A localização das fontes de dados ocorreu em biblioteca convencional e em sistemas de busca na internet, como LILACS e SCIELO, no período de abril a junho de 2013. Foram utilizadas as seguintes palavras-chave: saúde da família, educação física, promoção da saúde; prevenção de doenças. Os resultados encontrados apontam que a importância da atuação do profissional de Educação Física no NASF é reconhecida, entretanto, sua inserção nas equipes multiprofissionais precisa ser consolidada, uma vez constatado seu papel fundamental na prevenção de doenças e promoção da saúde.
\end{abstract}

Descritores: Saúde da Família; Educação Física; Promoção da Saúde; Prevenção de Doenças.

\section{The integration of professional physical education in multidisciplinary teams of family health strategy}

\begin{abstract}
The present study aimed to reflect on the importance of multidisciplinary teams in the Family Health Strategy, focusing on the importance of physical education professional. The methodology consisted of literature searches, based on the Ministerial Decree 154 of January 2008 that created the Centers of Support for Family Health (CSFH) and guidelines. The location of the data sources occurred in conventional library systems and web search, as LILACS and SciELO, in the period April-June 2013. The following key words were used: family health, physical education, health promotion; prevention of diseases. The results point to the importance of the role of physical education professional in Centers of Support for Family Health is recognized, however, it is necessary to consolidate their involvement in multidisciplinary teams, as it plays a key role in disease prevention and health promotion.
\end{abstract}

Descriptors: Family Health; Physical Education; Health Promotion; Disease Prevention.

\footnotetext{
${ }^{1}$ Bacharel em Enfermagem pela Universidade de Santa Cruz do Sul (UNISC), Santa Cruz do Sul, RS, Brasil.

${ }^{2}$ Especialista em Atividade Física, Desempenho Motor e Saúde pela Universidade Federal de Santa Maria (UFSM), Santa Maria, RS, Brasil.

${ }^{3}$ Doutora em Serviço Social pela Pontifícia Universidade Católica do Rio Grande do Sul (PUCRS), Porto Alegre, RS, Brasil.

${ }^{4}$ Mestre em Psicologia Clínica pela Pontifícia Universidade Católica de Campinas (PUCCAMP), Campinas, SP, Brasil.

${ }^{5}$ Doutora em Atividade Física e Saúde pela Faculdade de Desporto da Universidade do Porto (FADEUP), Porto, Portugal.

${ }^{6}$ Graduada em Licenciatura em Educação Física pela Universidade Federal de Santa Maria (UFSM), Santa Maria, RS, Brasil.
} 


\section{Introdução}

Atualmente, o ideário de promoção da saúde reconhece a natureza multifatorial da saúde, advoga sua desmedicalização, enfatiza o envolvimento comunitário e incorpora a ideia de educação para a saúde. ${ }^{1}$ Dessa forma, a saúde é definida não apenas como ausência de doenças, mas como uma condição humana com dimensões físicas, sociais e psicológicas, associados com a capacidade de apreciar a vida e saber enfrentar os desafios do cotidiano. ${ }^{1}$

O Programa Saúde da Família foi uma estratégia criada pelo Governo Federal em 1994, para implementar a atenção básica e contribuir para a qualidade de vida da população, considerando que a atenção primária é fundamental para que os cuidados em saúde alcancem grande parte da população. ${ }^{2}$ Pensar em saúde de forma ampliada, com vistas a prestar atendimento integral ao núcleo familiar, faz parte da formação do profissional da área da saúde. Esse atendimento diz respeito à promover a saúde do auto cuidado, com ações que contemplem princípios sociais, formativos e educativos. ${ }^{3}$ Trabalhar em equipe multiprofissional, como busca a Estratégia Saúde da Família, permite uma melhora na qualidade da assistência e o desenvolvimento de ações que visam mudanças na prática de saúde. ${ }^{2}$

Neste contexto, deve ser reconhecida a atuação de diversos profissionais, entre eles, o profissional de Educação Física junto aos Núcleos de Apoio à Saúde da Família (NASF) com o objetivo de melhorar a saúde e qualidade de vida da população. Assim, os campos de intervenção desse profissional vão além do aperfeiçoamento da aparência física, estando associados à melhora da saúde física e mental. ${ }^{4} \mathrm{~A}$ partir do reconhecimento do profissional de Educação Física como profissional da área da saúde, o presente estudo objetivou refletir sobre a importância de equipes multiprofissionais na Estratégia Saúde da Família, com enfoque no profissional de Educação Física.

\section{Metodologia}

A metodologia adotada consistiu de pesquisas na literatura, tendo como base a Portaria Ministerial n ${ }^{0} 154$ de 24 de janeiro de 2008, que criou os Núcleos de Apoio à Saúde da Família (NASF) e suas diretrizes. ${ }^{2}$ A localização das fontes ocorreu em biblioteca convencional e em sistemas de busca na internet, como Literatura Latino-Americana e do Caribe de Informação em Ciências da Saúde (LILACS) e Scientific Electronic Library Online (SciELO) no período de abril a junho de 2013. Foram utilizadas as seguintes palavras-chave: saúde da família, educação física, promoção da saúde; prevenção de doenças. Essas bases incluem informações e referências sobre a literatura internacional e científicotécnica da América Latina e do Caribe, sendo componente da Biblioteca Virtual em Saúde. As obras pesquisadas foram publicadas entre 1997 a 2012.

\section{Sistema Único de Saúde e Promoção da Saúde}

A Organização Mundial da Saúde (OMS) passou a conceituar saúde, a partir de 1949, como sendo "um completo bem estar físico, mental e social e não apenas ausência de doença". ${ }^{5}$ Nessa perspectiva, em 1988, foi estabelecida pela Constituição Brasileira uma nova formulação política e organizacional para o reordenamento dos serviços e ações de saúde.

A primeira Lei -n0 8.080- Lei Orgânica da Saúde, ${ }^{6}$ foi promulgada em 1990 e dispõem em Art. $2^{\circ}$ que "a saúde é um direito fundamental do ser humano, devendo o Estado prover as condições indispensáveis ao seu pleno exercício". Surge, então, o Sistema Único de Saúde (SUS), que segue a mesma doutrina, assim como os mesmos princípios organizativos em todo território nacional, sob três esferas autônomas de governo federal, estadual e municipal.

O SUS, tido como um novo sistema de saúde e que está em constante estruturação, é norteado por princípios doutrinários, entre eles, a Universalidade que prevê a garantia de atenção à saúde por parte do sistema, a todo e qualquer cidadão, da Eqüidade que assegura ações e serviços de todos os níveis de acordo com a complexidade que cada caso requeira, more o cidadão onde morar e a Integralidade em que as ações de promoção, proteção e recuperação da saúde não podem ser compartimentalizadas. ${ }^{5}$ Os princípios norteadores do SUS representam uma das maiores conquistas na efetivação da saúde em prol dos cidadãos. Contudo, a sua aplicação ainda não foi integralmente otimizada, uma vez que, inegavelmente, fazemos parte de um país numeroso com regiões de necessidades e realidades bastante diferenciadas, circunstâncias que, na prática, representam entraves para a sua efetiva aplicabilidade. ${ }^{5}$

O Sistema Único de Saúde enfrenta dificuldades para financiar a demanda crescente e, também, o alto custo das ações desenvolvidas em saúde. Esse sistema foi criado recentemente, sendo que os registros acerca de sua prática não 
contam mais do que duas décadas ${ }^{6}$, ainda assim é considerado um dos principais sistemas de saúde do mundo, o que demonstra sua eficácia. ${ }^{5}$

As políticas públicas de saúde no Brasil são regulamentadas segundo os preceitos do Sistema Único de Saúde (SUS), que apresenta como seu eixo norteador a Atenção Primária em Saúde (APS). Este modelo de atenção visa intervir nos determinantes sociais de saúde e possibilita uma compreensão ampliada do processo saúde/doença. ${ }^{2} \mathrm{O}$ foco do novo modelo de atenção à saúde está voltado para a promoção da saúde, o que torna-se evidente pela aprovação da Política Nacional de Promoção da Saúde (PNPS) que ratificou sua institucionalização no SUS. ${ }^{7}$

Declarações nacionais e internacionais ${ }^{8,9,10}$ vem relatando a importância da promoção da saúde, como forma de melhorar a qualidade de vida da população, além de legitimar a saúde como direito de todos e dever do estado. ${ }^{6}$ Nesse contexto, destaca-se a Primeira Conferência Internacional sobre Promoção da Saúde, realizada em Ottawa, Canadá $(1986)^{9}$, onde foram propostas estratégias para a melhoria da saúde e qualidade de vida, enfatizando a importância da participação da comunidade nesse processo. ${ }^{11}$

Portanto, a promoção da saúde parte de uma concepção ampla do processo saúde/doença e de seus determinantes. Esse conceito está associado a um conjunto de valores, como vida, saúde, solidariedade, equidade e cidadania. ${ }^{12,13}$ Refere-se também, a ações voltadas a implementação de políticas públicas e tecnologias e pressupõe a interação entre o setor sanitário e os demais setores da sociedade, entre o indivíduo e seu meio, produzindo uma rede de corresponsabilidade pelo bem-estar global. ${ }^{12,13}$

No âmbito das políticas públicas, a Política Nacional de Promoção da Saúde (PNPS) tem como objetivo central

Promover a qualidade de vida e reduzir a vulnerabilidade e riscos à saúde relacionados aos seus determinantes e condicionantes - modos de viver, condições de trabalho, habitação, ambiente, educação, lazer, cultura, acesso a bens e serviços essenciais. ${ }^{14}$

Dentre as suas diretrizes estão: o estímulo às ações intersetoriais; o fortalecimento da participação social e 0 incentivo a pesquisa em promoção da saúde. Destaca-se ainda que a PNPS propõem ações específicas para a prática corporal e atividade física na rede básica de saúde e na comunidade. ${ }^{14}$ Dessa forma, fica evidente a importância das práticas corporais e atividade física na promoção da saúde, constituindo um dos sete eixos temáticos de atuação da PNPS. Através da inserção do profissional de educação física na Estratégia de Saúde de Família torna-se possível capacitar a comunidade para melhorar sua qualidade de vida, considerando suas necessidades e o contexto cultural do qual fazem parte. ${ }^{15}$

\section{A Estratégia de Saúde da Família}

O Ministério da Saúde do Brasil escolheu como estratégia de reorientação para o modelo assistencial do SUS, a partir da atenção básica, a Saúde da Família, com objetivo de definir responsabilidades entre os serviços de saúde e a população. A implantação do Programa de Agentes Comunitários de Saúde (PACS), em junho de 1991, foi o passo inicial para essa estratégia. No ano de 1994, formaram-se as primeiras equipes completas onde um agente de saúde atuava com aproximadamente 575 pessoas. Já em 2007, o Brasil contava com 27.324 equipes implantadas em 5.125 municípios, atendendo $46,6 \%$ da população brasileira. ${ }^{5}$

Entre os objetivos da Estratégia de Saúde da Família, estão a prática de saúde humanizada por meio do vínculo entre profissionais de saúde e a população, a prestação de assistência integral e com resolutividade às necessidades da população, a intervenção sobre os fatores de risco aos quais a população está exposta, contribuindo para democratização do conhecimento do processo saúde/doença e fazendo com que a saúde seja reconhecida como um direito de cidadania. ${ }^{6}$

A Unidade de Saúde da Família é o primeiro contato com os serviços de saúde, sendo considerada como a "porta de entrada" de um sistema hierarquizado e regionalizado de saúde e fundamental para organização da referência e contra referência para os diferentes níveis do sistema. ${ }^{16}$ As equipes atuantes são compostas por profissional médico, enfermeiro, auxiliar de enfermagem, agentes comunitários de saúde, profissionais de saúde bucal e cirurgião-dentista. Cada equipe de Saúde da Família é capacitada para identificar a realidade das famílias pelas quais é responsável e, juntamente com a comunidade, elaborarem um plano que vise enfrentar os principais fatores categóricos no processo saúde/doença. ${ }^{16}$

O Ministério da Saúde criou, amparado na Portaria GM no 154, de 24 de Janeiro de 2008², o Núcleo de Apoio à Saúde da Família - NASF, visando apoiar a inserção da Estratégia Saúde da Família na rede de serviços e ampliar a abrangência, metas das ações e a resolutividade da Atenção Primária. O NASF deve ser constituído por equipes compostas por profissionais de diferentes áreas de conhecimento que atuem em parceria com os profissionais das 
Equipes Saúde da Família - ESF, compartilhando as práticas em saúde nos territórios sob responsabilidade das ESF, e atuando no apoio às equipes e na unidade em que o NASF está cadastrado. Possui como enfoque a promoção da saúde e o cuidado à população, procurando responder aos novos e antigos desafios da morbidade dos brasileiros, ampliando a oferta das práticas integrativas e ofertando a melhor tecnologia disponível para algumas das doenças crônicas. ${ }^{15}$

O NASF está classificado em duas modalidades, sendo NASF 1 composto por, no mínimo, cinco das profissões de nível superior, vinculado de 08 a 20 Equipes Saúde da Família, como: psicólogo, assistente social, farmacêutico, fisioterapeuta, fonoaudiólogo, profissional de educação física, nutricionista, terapeuta ocupacional, médico ginecologista, homeopata, acupunturista, pediatra e médico psiquiatra.

O NASF 2 deverá ser composto por três profissionais de nível superior, de ocupações não-coincidentes vinculado a 03 Equipes Saúde da Família, sendo eles: assistente social, profissional de educação física, farmacêutico, fisioterapeuta, fonoaudiólogo, nutricionista, psicólogo e terapeuta ocupacional. ${ }^{2} \mathrm{~A}$ Portaria $\mathrm{GM}^{2} \mathrm{n}^{0}$ determina que deve ser definida pelos gestores municipais a composição de cada um dos NASF, conforme critérios de prioridade a partir de cada necessidade local e, também, da disponibilidade dos profissionais de cada uma das diferentes ocupações.

Desse modo, é notável a existência de um regramento específico que ampara a atuação de diferentes profissionais visando à prevenção, promoção e reabilitação da saúde. Porém, neste ponto foi atribuído um critério subjetivo, ou seja, ficaria sob responsabilidade do gestor municipal a averiguação das necessidades, possibilidades e adequações de cada município.

\section{Equipes multiprofissionais na Estratégia Saúde da Família}

O trabalho em equipe, embora não seja exclusividade da Estratégia Saúde da Família, é considerado um dos pilares para a mudança do atual modelo hegemônico em saúde, com interação constante de diferentes profissionais, conhecimentos e habilidades para que o cuidado do usuário seja o imperativo ético-político que organiza a intervenção técnico-científica. ${ }^{16} \mathrm{~A}$ importância deste trabalho na ESF é ressaltada, principalmente, pelo aspecto de integralidade nos cuidados de saúde. Considerado um dos princípios doutrinários do Sistema Único de Saúde, a integralidade revestese, no decorrer dos anos 90, e principalmente nesse início de século, de uma importância estratégica ímpar para a consolidação de um novo modelo de atenção à saúde no Brasil. ${ }^{17}$

Nesse contexto, o diálogo na busca do consenso constitui-se como elemento imprescindível para o bom desenvolvimento do trabalho em equipe. Além disso, é importante considerar que uma equipe é composta por pessoas com especificidades próprias como gênero, inserção social, tempo e vínculo de trabalho, experiências profissionais e de vida, diferenças salariais e interesses próprios. Essas diferenças exercem influência sobre o processo de trabalho, pois estão presentes no agir de cada profissional. ${ }^{17}$

Os membros da equipe multiprofissional da ESF possuem funções específicas e devem atuar em uma perspectiva interdisciplinar. Ressalta-se que os agentes comunitários de saúde são pertencentes à comunidade onde atuam, sendo fundamentais na comunicação entre equipe e comunidade. Além disso, são orientados pelo médico e enfermeiro da Unidade de Saúde, fazendo visitas aos domicílios e anotando as informações necessárias à saúde da comunidade, bem como dedicando oito horas por dia ao seu trabalho. ${ }^{5}$

Ao técnico e auxiliar de enfermagem cabem, sob a supervisão do enfermeiro, realizar procedimentos regulamentados no exercício de sua profissão tanto na Unidade de Saúde quanto em domicílio ou na comunidade, educação em saúde e educação permanente. ${ }^{5}$ Junto aos agentes comunitários de saúde atua na identificação das famílias de risco e nas visitas domiciliares, participa das discussões e das organizações do processo de trabalho da Unidade de Saúde. ${ }^{5}$

O enfermeiro possibilita atividades de educação permanente, gerenciamento e a avaliação das atividades do grupo. Atende a saúde dos indivíduos e famílias cadastradas realizando a consulta de enfermagem, procedimentos, atividades em grupo e, conforme protocolos, solicitando exames complementares, prescrevendo medicações e gerenciando insumos e encaminhando usuários a outros serviços. ${ }^{18}$

O profissional médico deve promover a saúde, prevenindo, diagnosticando e tratando doenças. Nesse sentido, para que possa atender à demanda dos indivíduos sob sua responsabilidade, precisa realizar atividades programadas e de atenção à demanda espontânea, de forma compartilhada, consultas clínicas e pequenos procedimentos cirúrgicos, quando indicado na Unidade de Saúde, no domicílio ou em espaços comunitários.$^{18} \mathrm{O}$ médico da família deve, em um trabalho conjunto com o enfermeiro, realizar e fazer parte das atividades de educação permanente dos membros da equipe e participar do gerenciamento dos insumos. ${ }^{18}$

O cirurgião-dentista atua em conjunto com o técnico em saúde bucal (TSB), definindo o perfil epidemiológico da população para o planejamento e a programação em saúde bucal, a fim de oferecer atenção individual e atenção coletiva voltadas à promoção da saúde e à prevenção de doenças bucais, de forma integral e resolutiva. ${ }^{18}$ 
Cada equipe de Saúde da Família é capacitada para conhecer a realidade das famílias pelas quais é responsável. Desse modo, tem o compromisso de promover a saúde e elevar a qualidade de vida da população, prestando assistência integral, efetiva e contínua, considerando todo o contexto biopsicossocial. Destaca-se ainda que a confiança transmitida à comunidade é extremamente necessária, pois o vínculo estabelecido entre a Unidade de Saúde e as famílias possibilita uma maior aderência dos indivíduos ao Programa e sua efetividade, assim como os diferentes olhares dos profissionais integrantes da equipe que geram maior impacto sobre os diversos fatores que interferem no processo saúde-doença. ${ }^{18}$

\section{A atuação do profissional de educação física na ESF}

O reconhecimento da importância da inserção do profissional de Educação Física na operacionalização de políticas públicas voltadas a promoção e prevenção em saúde vem crescendo nas últimas décadas. Estudos comprovam que a prática regular de atividades físicas e a adoção de um estilo de vida ativo são fundamentais para a prevenção primária e tratamento de inúmeras doenças crônicas não transmissíveis, principalmente as relacionadas às doenças cardiovasculares e 0 câncer. ${ }^{19}$ Está associada também a prevenção ou a redução da osteoporose, melhora da capacidade funcional e bem-estar psicossocial, além de reduzir o estresse, a ansiedade e a depressão. ${ }^{20}$

A relação entre a prática regular de atividades físicas e melhoras nos padrões de saúde está estabelecida na literatura. Ainda assim, a prevalência de sedentarismo é elevada em diversos países. ${ }^{4} \mathrm{~A}$ Organização Mundial da Saúde ${ }^{20}$ recomenda a prática de 30 minutos de atividade física em cinco ou mais dias por semana, entretanto essa recomendação não é atingida por grande parcela da população. ${ }^{22}$ Portanto, o sedentarismo pode ser considerado um problema de saúde pública, tornando-se necessária a ampliação de políticas públicas de saúde por meio da inclusão do profissional de Educação Física, com o objetivo de promover hábitos saudáveis de vida na população.

A inserção do Profissional de Educação Física no NASF é de fundamental importância pelos aspectos físicos, cognitivos e sociais desenvolvidos por ele, promovendo melhora da qualidade de vida da população. Atuando junto à equipe multiprofissional do Núcleo de Apoio à Saúde da Família, o profissional de Educação Física amplia a abrangência da atenção básica como responsável pelas ações de atividades físicas e práticas corporais. ${ }^{23}$

Dentro da perspectiva de que as áreas estratégicas associadas ao NASF não se remetem à atuação específica e exclusiva de uma categoria profissional, o processo de trabalho do profissional de educação física deve ser caracterizado por ações compartilhadas, visando uma intervenção interdisciplinar. ${ }^{24}$ Portanto, a integração do Profissional de Educação Física deve ampliar e fortalecer as intervenções das equipes de saúde. Nesse contexto, o trabalho em equipe na saúde acontece na presença de profissionais com distintas formações na área e delineia-se pela capacidade que esses diferentes profissionais têm de produzir saúde. ${ }^{25}$ Dessa forma, a saúde deve ser pensada de forma ampliada com vistas a prestar atendimento integral aos indivíduos, através da formação de coletivos multiprofissionais.

As intervenções do profissional de Educação Física do NASF devem ser dirigidas a grupos-alvo, de diversas faixas etárias, portadores de diferentes condições corporais e/ou com necessidades de atendimentos especiais, junto à equipe multiprofissional do NASF. ${ }^{23}$ Além disso, o profissional deve conhecer as características do local e da população que the é referenciada, para atuar de acordo com suas necessidades. ${ }^{2}$

A Educação Física, vem se afirmando como atividade imprescindível à promoção da saúde e prevenção de doenças, nesse sentido, é de suma importância o entendimento das diferenças dessas práticas. As medidas de promoção da saúde não se dirigem a uma doença ou desordem específica, elas objetivam melhorar a saúde e o bem-estar de forma geral. ${ }^{11}$ Para tanto, é necessário que ocorra uma transformação das condições de vida e de trabalho que conformam a estrutura subjacente aos problemas de saúde, demandando uma abordagem intersetorial. ${ }^{11}$

Já as ações preventivas caracterizam-se por intervenções que buscam evitar o surgimento de doenças específicas, devem ser estruturadas mediante a divulgação de informação científica e de recomendações normativas de mudanças de hábitos. Essas ações tem o objetivo de diminuir a incidência e prevalência de doenças infecciosas e degenerativas, além de outros agravos específicos. ${ }^{11}$

A diferenciação entre os conceitos de promoção e prevenção da saúde é importante, pois possibilita a ampliação do olhar do profissional de Educação Física, não ficando limitado a ações focadas na doença, mas também aos aspectos positivos da saúde.

A inserção de um programa de práticas corporais/atividade física direcionada a população, como uma visão contemporânea, está apoiada em processos educativos e à ações de integração e socialização dos indivíduos. , $^{126}$ 


\section{Considerações Finais}

O Sistema Único de Saúde está em constante construção, passivo a novos programas e projetos e, nesse contexto, o novo paradigma de saúde, marcado pela promoção da saúde, abre um campo para atuação do Profissional de Educação Física nos serviços de saúde pública. Dessa forma, o profissional dessa área inserido no serviço de Atenção Básica do SUS deve estimular mudanças de atitude para a adoção de um estilo de vida saudável, levando em consideração as necessidades da população e o contexto na qual está inserida.

Ressalta-se ainda que o processo de trabalho do educador físico deve ser caracterizado por ações compartilhadas, visando uma intervenção interdisciplinar, ampliando e fortalecendo as intervenções das equipes de saúde. Portanto, é reconhecida a importância da atuação do profissional de Educação Física no NASF, entretanto, é preciso consolidar sua inserção nas equipes multiprofissionais. Para tanto, recomendam-se estudos referenciando os diversos aspectos de suas ações, uma vez que esta é uma área de atuação recente.

\section{Referências Bibliográficas}

1. Oliveira EM, Spiri WM. Programa Saúde da Família: a experiência de equipe multiprofissional. Departamento de Enfermagem. Rev Saúde Pública. 2006;40(4):727-33.

2. Ministério da Saúde, Cria os Núcleos de Apoio à Saúde da Família - NASF. Portaria n. 154, de 24 de janeiro de 2008. Brasilia (DF); 2008.

3. Rocha VM, Centurião $\mathrm{CH}$. Profissionais da saúde: formação, competência e responsabilidade social. In: Fraga $A B$, Wachs F. (Org.) Educação Física e Saúde Coletiva: políticas de formação e perspectivas de intervenção. Porto Alegre: UFRGS, 2007:17-31.

4. Bauman AF. Updating the evidence that physical activity is good for health: an epidemiological review. J Sci Med Sport. 2004;7(1):6-19.

5. Costa EMA, Carbone MH. Saúde da Família: Uma abordagem multidisciplinar. 2. Ed. Rio de Janeiro: Rubio;2009.

6. Lei Orgânica da Saúde n. 8.080, de 30 de setembro de 1990. Brasília (DF);1990.

Ministério da Saúde (BR). Saúde da Família: uma estratégia para a reorientação do modelo assistencial. Brasília (DF); 1997. 7. Malta DC, Castro AM, Gosch CS, et al. A Política Nacional de Promoção da Saúde e a agenda da atividade física no contexto do SUS. Epidemiol Serv Saúde. 2009;18;79-86.

8. Organização Mundial da Saúde. Declaração de Alma-Ata, 1978.

9. Organização Mundial da Saúde. Carta de Ottawa, 1986.

10. Organização Mundial da Saúde. Declaração de Sundsvall, 1991.

11. Czeresnia D, Freitas CM. Promoção da saúde: conceitos, reflexões, tendências. 2. Ed. Rio de Janeiro: Fiocruz;2009. 12. Moretti AC, Almeida V, Westphal MF, Bógus CM. Práticas corporais/atividade física e políticas públicas de Promoção da Saúde. Saúde Soci. 2009;18(2):346-354.

13. Buss PM. Health promotion and quality of life. Ciênc Saúde Coletiva. 2000;5(1):163-177, 2000.

14. Ministério da Saúde (BR), Secretaria de Vigilância em Saúde. Secretaria de Atenção à Saúde. Política Nacional de Promoção da Saúde. Brasília (DF); 2010.

15. Scabar TG, Pelicioni AF, Pelicioni MCF. Atuação do profissional de Educação Física no Sistema Único de Saúde: uma análise a partir da Política Nacional de Promoção da Saúde e das Diretrizes do Núcleo de Apoio à Saúde da Família (NASF). J Health Sci Inst. 2012;30(4):411-8.

16. Figueiredo, EN. A estratégia saúde da família na atenção básica do SUS. Universidade Federal de São Paulo-UNESPE, 2012. 17. Araújo MBS, Rocha PM. Trabalho em equipe: um desafio para a consolidação da estratégia de saúde da família. Cienc Saúde Coletiva. 2007;12(2):455-464.

18. Ministério da Saúde (BR), Departamento de Atenção Básica. Guia prático do programa de saúde da família. Brasília (DF); 2001. 19. Andersson J, Jansson JH, Hellsten G, Nilsson TK, Hallmans G, Boman K.. Effects of heavy endurance physical exercise on inflammatory markers in non-athletes. Atheroscler. 2010,209:601-605.

20. Ministério da Saúde (BR). Inquérito domiciliar sobre comportamentos de risco e morbidade referida de doenças e agravos não transmissíveis. Brasília (DF); 2003. 
21. World Health Organization. Global recommendations in physical activity for health. Geneva: World Health Organization, 2010.

22. Hallal PC, Dumith SC, Bastos JP, Reichert FF, Siqueira FV, Azevedo MR. Evolution on the epidemiological research on physical activity in Brazil: a systemic review. Rev Saúde Pública. 2007;41(3):453-460.

23. Conselho Federal de Educação Física. Intervenção do Profissional de Educação Física e respectivas competências e define os seus campos de atuação profissional. Resolução n046/2002, Rio de Janeiro;2002.

24. Carvalho YM, Freitas FF. Atividade física, saúde e comunidade. Cad Saúde Coletiva. 2006;14(3):489-506.

25. Bonaldi $C$. $O$ trabalho em equipe como dispositivo de integralidade: experiências cotidianas em quarto localidades brasileiras. In: Pinheiro B, Mattos R, Barros, MAB (Org.). Trabalho em equipe sob o eixo da integralidade: valores, saberes e práticas. Rio de Janeiro: Abrasco;2007.

26. Zioni F, Westphal MF. $O$ enfoque dos determinantes sociais de saúde sob o ponto de vista da teoria social. Saúde e Soci. 2007;16(3):26-34.

\section{Laísa Xavier Schuh}

Endereço para correspondência - Rua Sete de Setembro, n 739 -Ap.13, Bairro: Centro, CEP:96508-011, Cidade: Cachoeira do Sul, RS, Brasil.

E-mail: lalaschuh@hotmail.com

Lattes: http://lattes.cnpq.br/5393873764467271

Caroline Brand - carolbrand@hotmail.com.br

Suzane Beatriz Frantz Krug - skrug@unisc.br

Edna Linhares Garcia - edna@unisc.br

Anelise Reis Gaya - anegaya@gmail.com

Maria Amélia Roth - necaroth@gmail.com

Enviado em 27 de agosto de 2013. Aceito em 30 de junho de 2014. 
